\title{
ARTICLE
}

\section{Discovery of new element, nihonium, and perspectives (PLENARY)}

\author{
Kosuke Morita $^{\mathrm{a}, \mathrm{b}}$, Kouji Morimoto ${ }^{\mathrm{a}}$, Daiya Kaji ${ }^{\mathrm{a}}$, Hiromitsu Haba ${ }^{\mathrm{a}}$ and Hisaaki Kudo ${ }^{\mathrm{a}, \mathrm{c}^{*}}$ \\ ${ }^{a}$ RIKEN, 2-1 Hirosawa, Wako, Saitama 351-0198, Japan; ${ }^{b}$ Kyushu University, Higashi-ku, Fukuoka 812-8581, Japan; ${ }^{c}$ Niigata \\ University, 8050 Ikarashi 2-no cho, Nishi-ku, Niigata, 950-2181, Japan
}

\begin{abstract}
A new element 113 was searched by the reaction of ${ }^{70} \mathrm{Zn}$ on ${ }^{209} \mathrm{Bi}$ using a gas-filled recoil ion separator at the RIKEN linear accelerator facility. Totally three decay chains due to ${ }^{278} 113$ were observed. The first and second chains consisted of four alpha decays from ${ }^{278} 113$ to ${ }^{266} \mathrm{Bh}$, and terminated by spontaneous fission of ${ }^{262} \mathrm{Db}$. The 3 rd chain consisted of 6 consecutive alpha decays down to ${ }^{254} \mathrm{Md}$. The decay properties of the corresponding members of the observed 3 decay chains were consistent with each other. The net irradiation time altogether was 576 days and the deduced production cross section of ${ }^{278} 113$ was $22^{+20}{ }_{-13} \mathrm{fb}$. The decay properties of ${ }^{266} \mathrm{Bh}$ were investigated by the reaction of ${ }^{23} \mathrm{Na}$ on ${ }^{248} \mathrm{Cm}$ to establish the cross reaction of ${ }^{278} 113$ decay chain. It was found that ${ }^{266} \mathrm{Bh}$ can be regarded as an anchor nuclide of the ${ }^{278} 113$ decay chain. The name nihonium and symbol $\mathrm{Nh}$ were proposed for the element 113, and they were approved by the International Union of Pure and Applied Chemistry. A new research program at RIKEN toward discovery of heaviest elements by hot fusion reactions are also briefly described.
\end{abstract}

Keywords: new element; Nihonium; cold-fusion reaction; gas-filled recoil ion separator; ${ }^{278} 113 ;{ }^{266} \mathrm{Bh} ; \mathrm{hot}$ fusion reactions

\section{Introduction}

New element synthesis is one of most exciting subjects in connection with an extension of the periodic table of the elements. Production experiments of very heavy elements were conducted at RIKEN by use of a gas-filled recoil ion separator, GARIS. So-called cold-fusion type reactions were employed for the production of heavy elements, because produced new nuclides can be connected to known nuclides by $\alpha$-decay chains. The productions of heavy elements by cold-fusion type reactions were reported by Hofmann et al. and the employed reactions were ${ }^{208} \mathrm{~Pb}\left({ }^{64} \mathrm{Ni}, \mathrm{n}\right){ }^{271} \mathrm{Ds}$ $(Z=110) \quad[1],{ }^{209} \mathrm{Bi}\left({ }^{64} \mathrm{Ni}, \quad \mathrm{n}\right){ }^{272} \mathrm{Rg} \quad(Z=111) \quad[1], \quad$ and ${ }^{208} \mathrm{~Pb}\left({ }^{70} \mathrm{Zn}, \mathrm{n}\right){ }^{277} \mathrm{Cn}(Z=112)[2,3]$.

After a series of experiments were performed to check the performance of GARIS, the confirmation experiments of ${ }^{271} \mathrm{Ds},{ }^{272} \mathrm{Rg}$, and ${ }^{277} \mathrm{Cn}$ were conducted with the same reaction combinations as those employed by Hofmann et al. The results showed that the syntheses of these nuclides were successfully performed and identified in an extremely low-background condition with a high collection efficiency [4-6]. As an extension of these works, experiments aimed at synthesizing an isotope of element 113 using ${ }^{70} \mathrm{Zn}$ on ${ }^{209} \mathrm{Bi}$ reaction were performed and totally three decay chains due to ${ }^{278} 113$ were observed [7-9].

*Corresponding author. Email: hkudo@sc.niigata-u.ac.jp
The decay properties of ${ }^{266} \mathrm{Bh}$ which is the descendant of ${ }^{278} 113$ were also examined by the reaction of ${ }^{23} \mathrm{Na}$ on ${ }^{248} \mathrm{Cm}$ in order to establish the cross reaction of ${ }^{278} 113$ decay chain [10].

A new research program toward discovery of heaviest elements by hot fusion reactions has been started at RIKEN. The production of $\mathrm{Lv}$ isotopes was examined by the reaction of ${ }^{48} \mathrm{Ca}$ on ${ }^{248} \mathrm{Cm}$ using GARIS [11] and a newly constructed gas-filled recoil ion separator (GARIS-II) which is designed for hot-fusion reactions was successfully used to produce ${ }^{283} \mathrm{Cn}$ by the reaction of ${ }^{48} \mathrm{Ca}$ on ${ }^{238} \mathrm{U}[12]$.

\section{Experimental}

\subsection{Production of ${ }^{278} 113$}

A ${ }^{70} \mathrm{Zn}$ ion beam of $352.7 \mathrm{MeV}$ was extracted from the up-graded RIKEN Linear Accelerator, RILAC. The beam energy was determined by measuring a magnetic rigidity in a $90^{\circ}$ bending magnet and by a time-of-flight method. The absolute accuracy was \pm 0.6 $\mathrm{MeV}$. The beam intensity was monitored by measuring elastically scattered projectiles by the targets with a PIN photo-diode mounted at $45^{\circ}$ with respect to the incident beam direction in a distance of $1.28 \mathrm{~m}$ from the target position. Typical beam intensity on target was $2.4 \times 10^{12}$ $\mathrm{s}^{-1}$.

Bismuth targets were prepared by a vacuum 
evaporation of metallic bismuth onto carbon backing foils of $30 \mu \mathrm{g} / \mathrm{cm}^{2}$ thickness. The thickness of the bismuth target was about $450 \mu \mathrm{g} / \mathrm{cm}^{2}$. The targets were covered by a $10 \mu \mathrm{g} / \mathrm{cm}^{2}$ thick carbon to protect the target from sputtering. Beam energy at the half-depth of targets was estimated to $349.1 \mathrm{MeV}$ using range and stopping power tables [13]. Sixteen targets were mounted on a rotating wheel of $30 \mathrm{~cm}$ in diameter. The wheel was rotated by $2000 \mathrm{rpm}$ during the irradiation.

The reaction products were separated in-flight from the beam by GARIS and were guided into a detection system placed at the focal plane of GARIS (see Figure 1). The separator was filled with helium gas at a pressure of $86 \mathrm{~Pa}$. The value of magnetic rigidity of GARIS was set to $2.09 \mathrm{Tm}$.

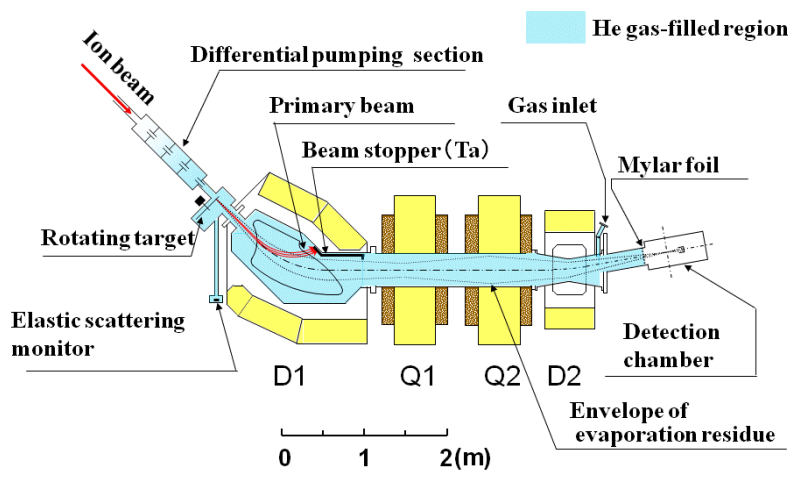

Figure 1. Schematic view of the experimental setup. D1 and D2 denote dipole magnets and Q1 and Q2 indicate quadrupole magnets.

The focal plane detection system consists of two sets of timing detectors and a silicon semiconductor detector box (SSD-box) placed downstream of the timing detectors. The timing detector is an assembly of a thin plastic foil and a micro-channel plate (MCP). Electrons emitted from the foil by impact of ions were bent by electrostatic field and injected to the MCP. Effective area of the timing detector is $78 \mathrm{~mm}$ in diameter. The SSD-box consists of five silicon detector plates. The size of each detector is $60 \mathrm{~mm} \times 60 \mathrm{~mm}$. One of the silicon detectors placed at the bottom of the SSD-box consists of 16 position-sensitive strip detectors (PSD). The size of each strip detector is $3.75 \mathrm{~mm} \times 60 \mathrm{~mm}$. The position resolution is about $\pm 1 \mathrm{~mm}$ in FWHM. The other four detectors (SSDs) are set to detect particles escaping from the PSD.

Rough mass numbers of incoming particles were estimated by the time signals together with the energy signals from PSD. The time signals were also used for the identification of decay events with an anticoincidence mode. The detection system was periodically checked by measuring the decays of the transfer products from the ${ }^{209} \mathrm{Bi}$ target. Counting rate of PSD was $2 \mathrm{~s}^{-1}$ at the typical beam intensity.

\subsection{Production of ${ }^{266} \mathrm{Bh}$}

For the production of ${ }^{266} \mathrm{Bh},{ }^{248} \mathrm{Cm}_{2} \mathrm{O}_{3}$ was used as a target material. The targets were prepared by electrodeposition onto $0.91 \mathrm{mg} / \mathrm{cm}^{2}$ thick titanium backing foils. The thickness of ${ }^{248} \mathrm{Cm}_{2} \mathrm{O}_{3}$ target was 350 $\mu \mathrm{g} / \mathrm{cm}^{2}$. Six pieces of the targets were mounted on a rotating wheel of $10 \mathrm{~cm}$ in diameter and the wheel was rotated at $1000 \mathrm{rpm}$ during the irradiation. The targets were irradiated with a ${ }^{23} \mathrm{Na}$ beam extracted from RILAC. The beam energies from RILAC were 126, 130, and 132 $\mathrm{MeV}$ which correspond to 121,124 , and $126 \mathrm{MeV}$, respectively, at the middle of the target.

The ${ }^{23} \mathrm{Na}$ beam was pulsed in two ways, micro and macro pulse structures. A purpose of the micro pulse structure was to prevent the irradiation of the target frame. A macro pulse structure was $3 \mathrm{~s}$ beam $\mathrm{ON}$ and $3 \mathrm{~s}$ beam OFF independently of the micro pulse structure. A logical AND signal of the micro and macro pulse structures was sent to the beam pulsing system of the accelerator. All the measurement was done only in the beam OFF period. A counting duty in the macro beam OFF period was $100 \%$, while that in the macro beam ON period was $45 \%$, which corresponds to the beam duty factor of $27.5 \%$. A typical beam intensity was 4.4 particle- $\mu \mathrm{A}$ in the micro beam $\mathrm{ON}$ period.

GARIS was used to separate evaporation residues (ERs) from the beam particles and other unwanted charged particles. GARIS was filled with helium gas at a pressure of $33 \mathrm{~Pa}$. The value of magnetic rigidity of GARIS was set at $2.07 \mathrm{Tm}$ for 132 and $130 \mathrm{MeV}$ beam energies, because this value of magnetic rigidity was known to give the highest yield of ${ }^{265} \mathrm{Sg}$ in the ${ }^{248} \mathrm{Cm}\left({ }^{22} \mathrm{Ne}, 5 \mathrm{n}\right){ }^{265} \mathrm{Sg}$ reaction. And the value of $2.19 \mathrm{Tm}$ was selected for $126 \mathrm{MeV}$ and a part of $132 \mathrm{MeV}$ to reduce a counting rate of the focal plane detector.

The detection system is about the same with the case of 113 experiment except for the timing detectors. Because the kinetic energy of ER is very low in the reaction of ${ }^{23} \mathrm{Na}$ on ${ }^{248} \mathrm{Cm}$, the timing detectors were removed. Energy calibration of the focal plane detectors was made using long-lived transfer products, ${ }^{248} \mathrm{Cf}$, ${ }^{252} \mathrm{Fm}$, and ${ }^{254} \mathrm{Fm}$ which were implanted in the PSD. A total counting rate of the focal plane detector was (5-10) $\mathrm{x} 10^{3} \mathrm{~s}^{-1}$ in the beam ON period, while that was $5-10 \mathrm{~s}^{-1}$ in the beam OFF period. Because of the high implantation rate to the PSD in the beam ON period, the PSD was gradually damaged in the course of the measurement and replaced three times during the experimental period of one and a half months.

We have done off-line analyses to search for mother-daughter correlation, i.e. $\alpha-\alpha$ and $\alpha$-SF event based on position in PSD, energy, and time difference. Because data only at beam OFF period were used, a signal of implantation of ER was not recorded. Therefore, time difference between ER implantation and its decay event was not obtained. An average counting rate of particles having energies of greater than $50 \mathrm{MeV}$ for each strip of the PSD was $2.7 \times 10^{-4} \mathrm{~s}^{-1}$. A counting rate in the positional window of $2 \mathrm{~mm}$ is calculated to be 
$9.3 \times 10^{-6} \mathrm{~s}^{-1}$. Considering a time window of $300 \mathrm{~s}$, a probability of an accidental correlation is calculated to be $2.8 \times 10^{-3}$ per event. This low value of the accidental probability was realized by using a combination of GARIS and PSD, which enables a low background $\alpha$ -SF correlation measurement compared with a gas-jet transport system.

\section{Results and discussion}

The first event of the candidate of ${ }^{278} 113$ was observed on July 23, 2004 [7]. The net irradiation time was 79 days and the total dose of ${ }^{70} \mathrm{Zn}$ was $1.7 \times 10^{19}$. The event consisted of an implantation signal in the PSD followed by four consecutive $\alpha$-decays and a spontaneous fission as a terminal signal. The measured positions of all six sequential signals were within the spatial resolution of the PSD. The observed energies and the time differences between decay signals are indicated in Figure 2 as a label of "2004". The energy was calibrated for $\alpha$-particles, so that the energy for the spontaneous fission may not be accurate because of a possible pulse height defect. The $\alpha$-particles of the 1 st and 4th events were detected only by the PSD whereas those of the 2nd and 3rd were partly measured by the PSD and partly by the SSD. The energy resolution measured by only the PSD was $39 \mathrm{keV}$ in full width at half maximum (FWHM) and that measured by both the detectors was $66 \mathrm{keV}$ in FWHM.

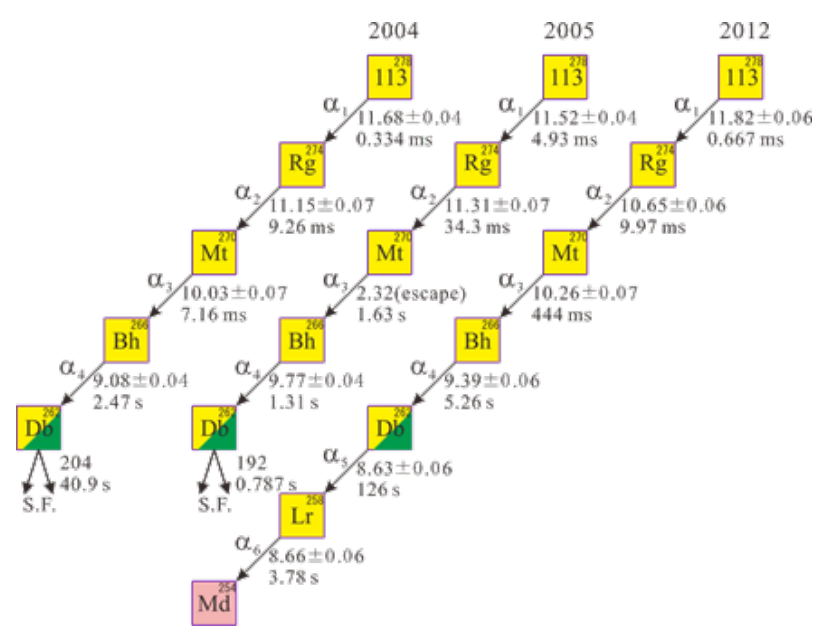

Figure 2. Observed $\mathrm{Z}=113$ decay chains. Values are $E_{\alpha}(\mathrm{MeV})$ and lifetimes.

For all the five decay events, no TOF signal registered from the timing detectors was associated. Considering the counting rate of decay events and the time difference, the probabilities of accidental coincidence between ER and individual decays were evaluated to be $2.9 \times 10^{-9}, 8.2 \times 10^{-8}, 1.4 \times 10^{-7}, 2.1 \times 10^{-5}$, and $9.5 \times 10^{-6}$, for $\alpha_{1}, \alpha_{2}, \alpha_{3}, \alpha_{4}$, and SF, respectively. An example of correlation analysis is shown in Figure 3. The energy spectra of the PSD signals with different gates are illustrated for the run in which the above mentioned event was observed. The duration of this run was 12.7 hours. The uppermost spectrum (a) indicates a singles spectrum without any gate and spectrum (b) is the same as (a) but expanded to the region of interest corresponding to decay-alpha energies. As the beamoriginated signals in PSD are considered to be coincident with the signals from the timing detector, signals without the timing detector are regarded as the decay events in PSD. Such signals are displayed in spectrum (c). If a position correlation with $\pm 1 \mathrm{~mm}$ and a limiting time window of $60 \mathrm{~s}$ are taken into account for the signals in spectrum (c), only the true decay events remain as shown in spectrum $(d)$.

The mass number of ER was roughly estimated to be $280 \pm 15$ from the data of TOF between the timing counters of 44.6 ns and the energy measured by the PSD of $36.75 \mathrm{MeV}$ using a method similar to that described in Ref. 5.

Total (High gain)

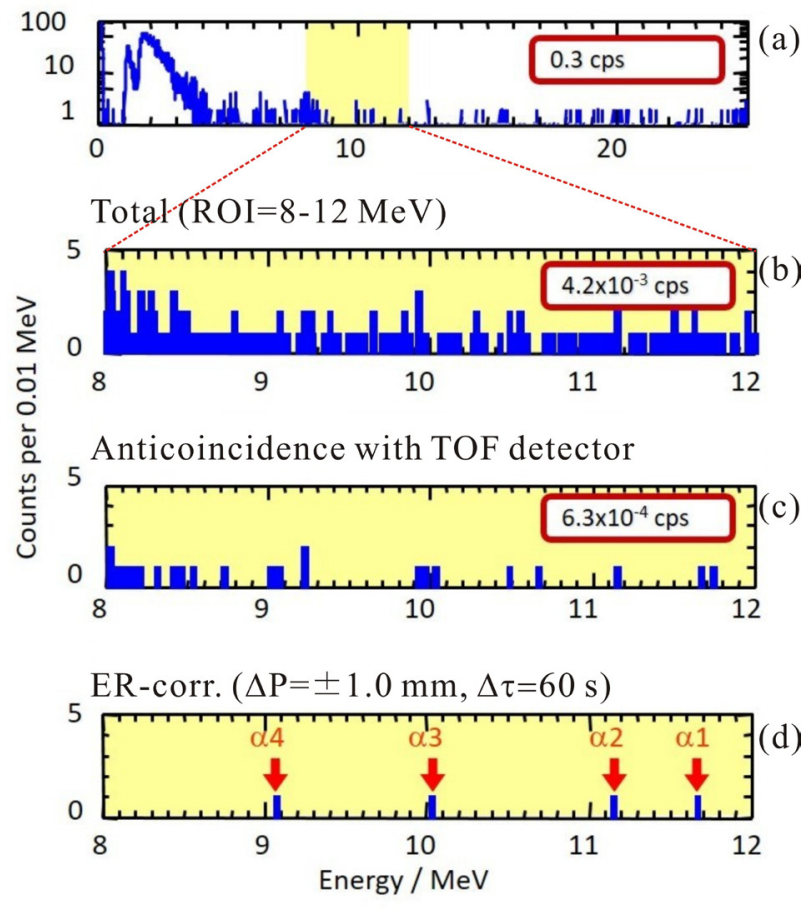

Figure 3. Energy spectra measured at one strip of PSD where the first event was observed. (a) singles spectrum without any gate;(b) the same as (a) but expanded to an energy region of interest;(c) spectrum of signals without TOF signals;(d) residual signals of (c) with a position gate $(\Delta \mathrm{P}= \pm 1 \mathrm{~mm})$ and a time gate $(\Delta \tau<60 \mathrm{~s})$.

The second candidate was observed on April 2, 2005 [8]. The net irradiation time was 161 days and the total dose of ${ }^{70} \mathrm{Zn}$ was $4.45 \times 10^{19}$. The second decay sequence is about the same with that observed in the first one, that is, four consecutive $\alpha$-decays followed by a spontaneous fission after the implantation of ER. The decay energies and the time differences between decay signals are 
indicated in figure 2 as a label of " 2005 ". The decay energy of the 3 rd $\alpha$-decay was measured only in part owing to the limited solid angle coverage $(85 \%)$ of the detector box. The mass number are almost the same with that of the first one, because the values of TOF data and the energies at the PSD are in good agreement with each other.

The third decay chain was observed on August 12, 2012 [9]. The observed decay chain consisted of six consecutive $\alpha$-decays after the implantation of an ER in the PSD. The associated $\alpha$-energies and the time intervals are summarized as "2012" in figure 2. The $\alpha$-decay mode was first observed for the fifth decay in the third chain, while the modes observed in the first and second decay chains were the SF decay. The observed $\alpha$-energy of $\mathrm{E}_{\alpha}=8.63 \pm 0.06 \mathrm{MeV}$ is in good agreement with the adopted values for ${ }^{262} \mathrm{Db}$ [14]. In the present decay chain, the sixth $\alpha$-decay $\left(\alpha_{6}\right)$ of $E_{\alpha}=8.66 \pm 0.06$ $\mathrm{MeV}$ was also observed with a decay time of $3.78 \mathrm{~s}$, which corresponds to $\mathrm{T}_{1 / 2}=2.6_{-1.1}^{+12} \mathrm{~s}$. These decay properties are in good agreement with the adopted properties for ${ }^{258} \mathrm{Lr}$ which is the $\alpha$-decay daughter of ${ }^{262} \mathrm{Db}$. The mass number of ER was roughly estimated to be $291 \pm 15$.

The experiment was designed to produce the isotope, ${ }^{278} 113$, by the one-neutron evaporation channel in the ${ }^{209} \mathrm{Bi}+{ }^{70} \mathrm{Zn}$ reaction. On the basis of a systematic study of the most probable reaction energies for the one-neutron evaporation channel in the ${ }^{208} \mathrm{~Pb}\left({ }^{64} \mathrm{Ni}\right.$, n) ${ }^{271} \mathrm{Ds}(Z=110)[4],{ }^{209} \mathrm{Bi}\left({ }^{64} \mathrm{Ni}, \mathrm{n}\right){ }^{272} \mathrm{Rg}(Z=111)[5]$, and ${ }^{208} \mathrm{~Pb}\left({ }^{70} \mathrm{Zn}, \mathrm{n}\right){ }^{277} \mathrm{Cn}(Z=112)[6]$ reactions, a reaction energy of $349.0 \mathrm{MeV}$ at the half-depth of the targets was adopted to produce ${ }^{278} 113$. The corresponding energy of the center of mass frame is $261.4 \pm 2.0 \mathrm{MeV}$, where \pm 2.0 $\mathrm{MeV}$ indicates the range of reaction energy due to the energy loss of the beam in the bismuth targets. The excitation energy of the compound nucleus, ${ }^{279} 113$, is calculated to be $14.1 \pm 2.0 \mathrm{MeV}$ using the predicted mass for a compound nucleus [15] and the experimental masses of the beam and target [16]. This excitation energy is considered to be too high for a radiative capture process (zero neutron emission channel) and too low for a two-neutron emission channel. The possibility of a one-proton evaporation channel leading to the product of ${ }^{278} 112$ is excluded by comparing the observed decay time of SF with that of ${ }^{262} \mathrm{Rf}$ which decays by $100 \%$ spontaneous fission with a half-life of $47 \pm 5 \mathrm{~ms}$ [17]. As a result, the members of the decay chains were assigned as ${ }^{278} 113,{ }^{274} \mathrm{Rg},{ }^{270} \mathrm{Mt},{ }^{266} \mathrm{Bh},{ }^{262} \mathrm{Db}$, and ${ }^{258} \mathrm{Lr}$.

The decay time distributions of individual generations in the three decay chains are shown in Figure 4, where the logarithm of the decay time is taken as the abscissa. It was found that the decay time of each generation closely follows a single exponential decay curve. This indicates that the observed decays originated from nuclides with the same mean life. The $\alpha$-decay energies of ${ }^{278} 113,{ }^{274} \mathrm{Rg},{ }^{270} \mathrm{Mt}$, and ${ }^{266} \mathrm{Bh}$ show some discrepancies among the three chains. However, the observation in the rather widely distributed decay energies of the decay chains of odd-odd nuclei is a natural feature due to the decays to many excited states in their daughters, and due to the possible summing effect of $\alpha$-energy with a conversion electron or $\gamma$-ray energy, which is emitted simultaneously with $\alpha$-decay inside the detector.

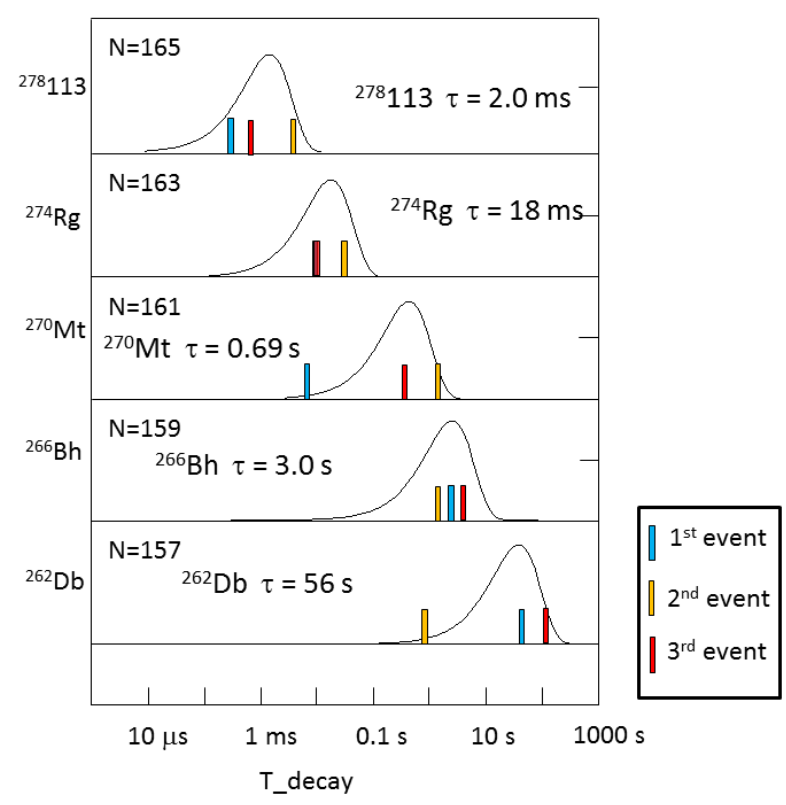

Figure 4. Decay time distributions of individual generations in the three decay chains.

The total beam dose was $1.4 \times 10^{20}$. Combining all three events, the production cross section of ${ }^{278} 113$ was determined to be $22^{+20}{ }_{-13} \mathrm{fb}$ with $1 \sigma$ error. The error includes only a statistical one. To deduce the cross section, the values of the transmission of the GARIS was assumed to be 0.8 .

In the off-line analysis of the ${ }^{266} \mathrm{Bh}$ experiment, the maximum correlation time was set to $300 \mathrm{~s}$ and the position window was set to $\pm 2 \mathrm{~mm}$ for the correlation events measured only in the PSD. We have assigned total of 32 correlations to true ones [10]. Four of the events are $\alpha_{1}-\alpha_{2}-\alpha_{3}$ correlations, one is an $\alpha_{1}-\alpha_{2}-S F$, nine are $\alpha_{1}-\alpha_{2}$ and eighteen are $\alpha_{1}$-SF correlations. Because ER cross sections of asymmetric reactions in the heaviest region exhibit a broad peak around the optimum energies, ${ }^{267} \mathrm{Bh}$, which is a product of the $4 \mathrm{n}$ evaporation channel of the reaction, could be produced simultaneously with ${ }^{266} \mathrm{Bh}$. We focused on these two isotopes in the analyses. The direct productions of ${ }^{262} \mathrm{Db}$ and ${ }^{263} \mathrm{Db}$, which are the $\alpha$-decay daughters of ${ }^{266} \mathrm{Bh}$ and ${ }^{267} \mathrm{Bh}$ by $\alpha 5 \mathrm{n}$ and $\alpha 4 \mathrm{n}$ evaporation channels, respectively, were not considered because the present bombarding energies were in the sub-barrier energy region. As a result of correlation analysis, 11 chains which contain $\alpha$-decay of ${ }^{262} \mathrm{Db}$ and 3 chains which include SF of ${ }^{262} \mathrm{Db}$ were conclusively assigned to ${ }^{266} \mathrm{Bh}$ decay. As for the half-life of ${ }^{266} \mathrm{Bh}$, we could only state 
that it is longer than $1 \mathrm{~s}$ because of the small counting statistics. The obtained decay properties are in agreement with the reported ones $[18,19]$ which were deduced from the different nuclear reactions. Thus, the decay properties of ${ }^{266} \mathrm{Bh}$ were well established by cross-reaction experiments and ${ }^{266} \mathrm{Bh}$ can be regarded as an anchor nuclide of ${ }^{278} 113$ decay chain. The observed decay chains from ${ }^{278} 113$ are connected to the anchor nuclide, ${ }^{266} \mathrm{Bh}$, and thus the production of ${ }^{278} 113$ are confirmed.

The discovery of a new element 113 was recognized by the fourth IUPAC/IUPAP Joint Working Party on the priority of claims to the discovery of new elements [20]. The name nihonium and symbol $\mathrm{Nh}$ were proposed for the element 113, and they were approved by the International Union of Pure and Applied Chemistry [21].

\section{Perspectives}

The isotope of the 113 th element, i.e., ${ }^{278} 113$, was produced in the ${ }^{209} \mathrm{Bi}\left({ }^{70} \mathrm{Zn}, \mathrm{n}\right){ }^{278} 113$ reaction and was unambiguously identified by the firm connection to the well-known daughter nuclides ${ }^{266} \mathrm{Bh},{ }^{262} \mathrm{Db}$, and ${ }^{258} \mathrm{Lr}$. But the production cross section of ${ }^{278} 113$ was very small as $22^{+20}-13 \mathrm{fb}$. If cross sections of heavier elements follow a trend of those for products of cold-fusion type reactions, cross sections of elements of $Z>118$ are expected to be quite small and it is practically not feasible to synthesize these elements by cold-fusion type reactions.

An alternative way to produce super heavy elements is based on so-called hot fusion reactions. The discoveries of elements 114 to 118 were successfully accomplished using this method by Oganessian et al. [22]. Along this line, the production of Lv isotopes by the reaction of ${ }^{48} \mathrm{Ca}$ on ${ }^{248} \mathrm{Cm}$ was performed by use of GARIS at RIKEN [11]. The isotopes of ${ }^{292} \mathrm{Lv}$ and ${ }^{293} \mathrm{LV}$ were successfully produced and the deduced cross sections of these isotopes are consistent with the reported values [22]. A transmission of GARIS was assumed to be $35 \%$ which was determined from the test reaction of ${ }^{208} \mathrm{~Pb}\left({ }^{48} \mathrm{Ca}, 2 \mathrm{n}\right){ }^{254} \mathrm{No}$.

As the performance of GARIS is most suitable for cold-fusion type reactions, a new gas-filled recoil ion separator GARIS-II was developed for the investigation of reaction products by hot-fusion reactions [23]. The schematic view of GARIS-II is shown in Figure $\mathbf{5}$ and some ion optical characteristics are tabulated in Table $\mathbf{1}$ in comparison with GARIS. A transmission of GARIS-II was measured for ${ }^{208} \mathrm{~Pb}\left({ }^{48} \mathrm{Ca}, 2 \mathrm{n}\right){ }^{254} \mathrm{No}$ and deduced to be $72 \%$ which is about twice of GARIS. By use of GARIS-II ${ }^{283} \mathrm{Cn}$ produced in the ${ }^{238} \mathrm{U}\left({ }^{48} \mathrm{Ca}, 3 \mathrm{n}\right)$ reaction was measured and two decay chains from ${ }^{283} \mathrm{Cn}$ were observed at the compound-nucleus excitation energy of 34.5 MeV [12]. The decay properties and the cross section of the observed decay chains are consistent with the reported ones [22].

The newly developed GARIS-II is a promising apparatus for the measurement of super heavy elements produced in actinide-based, hot-fusion reactions.

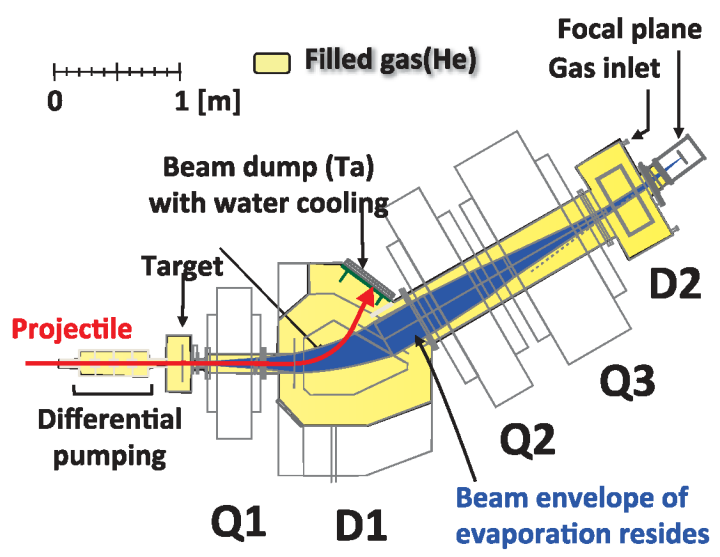

Figure 5. Schematic view of a newly constructed gas-filled recoil ion separator (GARIS-II). D1 and D2 denote dipole magnets and Q1, Q2, and Q3 indicate quadrupole magnets.

Table 1. Ion optical characteristics of GARIS-II and GARIS

\begin{tabular}{|l|l|l|}
\hline & GARIS-II & GARIS \\
\hline Configuration & QvDQhQvD & DQvQhD \\
\hline Ang. acceptance (X) (mrad) & \pm 47 & \pm 61 \\
\hline Ang. acceptance (Y) (mrad) & \pm 110 & \pm 57 \\
\hline Solid angle (msr) & 18.5 & 12.2 \\
\hline Total path length (m) & 5.06 & 5.76 \\
\hline Maximum Bq (Tm) & 2.43 & 2.16 \\
\hline Dispersion (mm/\%) & 19.3 & 9.7 \\
\hline
\end{tabular}

\section{References}

[1] S. Hofmann, V. Ninov, F. P. Heßberger, P. Armbruster, H. Folger, G. Münzenberg, H. J. Schött, A. G. Popeko, A. V. Yeremin, A. N. Andreyev, S. Saro, R. Janik and M. Leino, The new element 111, Z. Phys. A350 (1995), pp. 281-282.

[2] S. Hofmann, V. Ninov, F. P. Heßberger, P. Armbruster, H. Folger, G. Münzenberg, H. J. Schött, A. G. Popeko, A.V. Yeremin, S. Saro, R. Janik and M. Leino, The new element 112, $Z$. Phys. A354 (1996), pp. 229-230.

[3] S. Hofmann, F.P. Heßberger, D. Ackermann, G. Münzenberg, S. Antalic, P. Cagarda, B. Kindler, J. Kojouharova, M. Leino, B. Lommel, R. Mann, A.G. Popeko, S. Reshitko, S. Śaro, J. Uusitalo and A.V. Yeremin, New results on elements 111 and 112, Eur. Phys. J. A14 (2002), pp. 147-157.

[4] K. Morita, K. Morimoto, D. Kaji, H. Haba, E. Ideguchi R. Kanungo, K. Katori, H. Koura, H. Kudo, T. Ohnishi, A. Ozawa, T. Suda, K. Sueki, I. Tanihata, H. Xu, A.V. Yeremin, A. Yoneda, A. Yoshida, Y.-L. Zhao and T. Zheng, Production and decay of the isotope ${ }^{271} \mathrm{Ds}(\mathrm{Z}=110)$, Eur. Phys. J. A21 (2004), pp. 257-263.

[5] K. Morita, K. Morimoto, D. Kaji, H. Haba, E. Ideguchi, R. Kanumgo, K. Katori, H. Koura, H. 
Kudo, T. Ohnishi, A. Ozawa, T. Suda, K. Sueki, I. Tanihata, H. Xu, A.V. Yeremin, A. Yoneda, A. Yoshida, Y.-L. Zhao and T. Zhen, Production and decay properties ${ }^{272} 111$ and its daughter nuclei, $J$. Phys. Soc. Jpn. 73 (2004), pp. 1738-1744.

[6] K. Morita, K. Morimoto, D. Kaji, T. Akiyama, S. Goto, H. Haba, E. Ideguchi, K. Katori, H. Koura, H. Kudo, T. Ohnishi, A. Ozawa, T. Suda, K. Sueki, F. Tokanai, T. Yamaguchi, A. Yoneda and A. Yoshida, Experiment on the synthesis of of an isotope ${ }^{277} 112$ by ${ }^{208} \mathrm{~Pb}+{ }^{70} \mathrm{Zn}$ reaction, J. Phys. Soc. Jpn. 76 (2007), 043201-1-5.

[7] K. Morita, K. Morimoto, D. Kaji, T. Akiyama, S. Goto, H. Haba, E. Ideguchi, R. Kanungo, K. Katori, H. Koura, H. Kudo, T. Ohnishi, A. Ozawa, T. Suda, K. Sueki, H. Xu, T. Yamaguchi, A. Yoneda, A and Yoshid,Y.L. Zhao, Experiment on the synthesis of element 113 in the reaction ${ }^{209} \mathrm{Bi}\left({ }^{70} \mathrm{Zn}, \mathrm{n}\right){ }^{278} 113, J$. Phys. Soc. Jpn. 73 (2004), pp. 2593-2596.

[8] K. Morita, K. Morimoto, D. Kaji, T. Akiyama, S. Goto, H. Haba, E. Ideguchi, K. Katori, H. Koura, H. Kikunaga, H. Kudo, T. Ohnishi, A. Ozawa, N. Sato, T. Suda, K. Sueki, F. Tokanai, T. Yamaguchi, A. Yoneda and A. Yoshida, Observation of second decay chain from ${ }^{278} 113$, J. Phys. Soc. Jpn. 76 (2007), 045001-1-2.

[9] K. Morita, K. Morimoto, D. Kaji, H. Haba, K. Ozeki, Y. Kudou, T. Sumita, Y. Wakabayashi, A. Yoneda, K. Tanaka, S. Yamaki, R. Sakai, T. Akiyama, S. Goto, H. Hasebe, M. Huang, T. Huang, E. Ideguchi, Y. Kasamatsu, K. Katori, Y. Kariya, H. Kikunaga, H. Koura, H. Kudo, A. Mashiko, K. Mayama, S. Mitsuoka, T. Moriya, M. Murakami, H. Murayama, S. Namai, A. Ozawa, N. Sato, K. Sueki, M. Takeyama, F. Tokanai, T. Yamaguchi and A. Yoshida, New result in the production and decay of an isotope, ${ }^{278} 113$, of the 113th element, J. Phys. Soc. Jpn. 81 (2012), 103201-1-4.

[10]K. Morita, K. Morimoto, D. Kaji, H. Haba, K. Ozeki, Y. Kudo, N. Sato, T. Sumita, A. Yoneda, T. Ichikawa, Y. Fujimori, S. Goto, E. Ideguchi, Y. Kasamatsu, K. Katori, Y. Komori, H. Koura, H. Kudo, K. Ooe, A. Ozawa, F. Tokanai, K. Tsukada, T. Yamaguchi and A. Yoshida, Decay properties of ${ }^{266} \mathrm{Bh}$ and ${ }^{262} \mathrm{Db}$ produced in the ${ }^{248} \mathrm{Cm}+{ }^{23} \mathrm{Na}$ Reaction, J. Phys. Soc. Jpn. 78 (2009), 064201-1-6.

[11]D. Kaji, K. Morita, K. Morimoto, H. Haba, M. Asai, K. Fujita, Z. Gan, H. Geissel, H. Hasebe, S. Hofmann, M. Huang, Y. Komori, L. Ma, J. Maurer, M. Murakami, M. Takeyama, F. Tokanai, T. Tanaka, Y. Wakabayashi, T. Yamaguchi, S. Yamaki and A. Yoshida, Study of the reaction ${ }^{48} \mathrm{Ca}+{ }^{248} \mathrm{Cm} \rightarrow$ ${ }^{296}$ Lv* $^{*}$ at RIKEN-GARIS, J. Phys. Soc. Jpn. 86 (2017), 034201-1-7.
[12]D. Kaji, K. Morimoto, H. Haba, Y. Wakabayashi, M. Takeyama, S. Yamaki, Y. Komori, S. Yanou, S. Goto and K. Morita, Decay measurement of ${ }^{283} \mathrm{Cn}$ produced in the ${ }^{238} \mathrm{U}\left({ }^{48} \mathrm{Ca}, 3 \mathrm{n}\right)$ reaction using GARIS-II, J. Phys. Soc. Jpn. 86 (2017), 085001-1-2.

[13]L.C. Northcliffe and R.F. Schilling, Range and stopping-power tables for heavy ions, Nucl. Data Tables, A7 (1970), pp. 233-463.

[14]R. Dressler, B. Eichler, D.T. Jost, D. Piguet, A. Türler, Ch. Düllmann, R. Eichler, H.W. Gäggeler, M. Gärtner, M. Schädel, S. Taut and A.B. Yakushev, Production of ${ }^{262} \mathrm{Db}(\mathrm{Z}=105)$ in the reaction ${ }^{248} \mathrm{Cm}\left({ }^{19} \mathrm{~F}, 5 \mathrm{n}\right)$, Phys. Rev. C59 (1999), pp. 3433-3436.

[15]W.D. Myers and W.J. Swiatecki, Nuclear properties according to the Thomas-Fermi model, Nucl. Phys. A601 (1996), pp. 141-167.

[16]G. Audi and A.H. Wapstra, The 1993 atomic mass evaluation: (I) Atomic mass table, Nucl. Phys. A565 (1993), pp. 1-65.

[17] L.P. Somerville, M.J. Nurmia, J.M. Nitschke, A. Ghiorso, E.K. Hulet, R.W. Lougheed, Spontaneous fission of rutherfordium isotopes, Phys. Rev. C31 (1985), pp. 1801-1815.

[18]P.A. Wilk, K.E. Gregorich, A. Türler, C.A. Laue, R. Eichler, V. Ninov, J.L. Adams, U.W. Kirbach, M.R. Lane, D.M. Lee, J.B. Patin, D.A. Shaughnessy, D.A. Strellis, H. Nitsche and D.C. Hoffman, Evidence for new isotopes of element 107: ${ }^{266} \mathrm{Bh}$ and ${ }^{267} \mathrm{Bh}$, Phys. Rev. Lett. 85 (2000), pp. 2697-2700.

[19]Z. Qin, X. Wu, H. Ding, W. Wu, W. Huang, X. Lei, Y. Xu, X. Yuan, B. Guo, W. Yang, Z. Gan, H. Fan, J. Guo, H. Xu and G. Xiao, Alpha decay properties of ${ }^{266}$ Bh, Nucl. Phys. Rev. 23 (2006), pp. 404-407, (Chinese journal in English).

[20]P.J. Karol, R.C. Barber, B.M. Sherrill, E. Vardaci and T. Yamazaki, Discovery of the elements with atomic numbers $\mathrm{Z}=113,115$ and 117 (IUPAC Technical Report), Pure Appl. Chem. 88 (2016), pp. 139-153.

[21]L. Öhrström and J. Reedijk, Names and symbols of the elements with atomic numbers 113, 115, 117 and 118 (IUPAC Recommendations 2016), Pure Appl. Chem. 88 (2016), pp. 1225 -1229.

[22]Yu.Ts. Oganessian and V.K. Utyonkov, Superheavy nuclei from ${ }^{48} \mathrm{Ca}$-induced reactions, Nucl. Phys. A944 (2015), pp. 62-98, and references therein.

[23]D. Kaji, K. Morimoto, N. Sato, A. Yoneda and K. Morita, Gas-filled recoil ion separator GARIS-II, Nucl. Instrum. Methods Phys. Res., Sect. B 317 (2013), pp. 311-314. 